\title{
Treatment of Osteoarthritis Secondary to Severe Coxa Vara with Modular Total Hip Arthroplasty
}

\author{
Bohan Zhang ${ }^{1,2, *}$ \\ Jingyang Sun ${ }^{1,2, *}$ \\ Yinqiao Du (iD ${ }^{2,3}$ \\ Junmin Shen ${ }^{2,4}$ \\ Tiejian $\mathrm{Li}^{1,2}$ \\ Yonggang Zhou $\mathbb{D}^{1-3}$ \\ 'Medical School of Chinese PLA, Beijing, \\ I00853, People's Republic of China; \\ ${ }^{2}$ Department of Orthopedics, The First \\ Medical Center of PLA General Hospital, \\ Beijing, 100853, People's Republic of \\ China; ${ }^{3}$ Senior Department of \\ Orthopedics, The Fourth Medical Center \\ of PLA General Hospital, Beijing, I00048, \\ People's Republic of China; ${ }^{4}$ Medical \\ School of Nankai University, Tianjin, \\ 30007I, People's Republic of China
}

*These authors contributed equally to this work
Correspondence: Yonggang Zhou Department of Orthopedics, The First Medical Center, Chinese People's

Liberation Army General Hospital, 28th

Fuxing Road, Haidian District, Beijing,

People's Republic of China

Tel +8613801287599

Email ygzhou30I@163.com
Objective: We aimed to demonstrate the methods of treatment for coxa vara with modular total hip arthroplasty (THA) and evaluate clinical and radiographic outcomes, and further survivorship at the midterm follow-up.

Methods: We retrospectively reviewed 33 patients (42 hips) who underwent modular THA for coxa vara deformity from May 2008 to December 2019. The clinical and radiographic results, including Harris Hip Score (HHS), leg length discrepancy (LLD), greater trochanteric height, femoral offset, abductor lever arm, stem alignment and limp, and complications, were evaluated.

Results: The follow-up time was mean $69.9 \pm 43.7$ months. Clinically, the HHS improved significantly $(\mathrm{p}<0.001)$ on average from $42.90 \pm 14.44$ points to $89.54 \pm 4.75$ points. The mean LLD decreased from $33.3 \pm 19.4 \mathrm{~mm}$ to $5.0 \pm 5.8 \mathrm{~mm}(\mathrm{p}<0.001)$, and 27 patients $(82 \%)$ thought that total equality of the lower limbs was obtained. Patients demonstrated diminished or no limping in $88 \%(29 / 33)$ of hips and a significant improvement of biomechanics. At the final follow-up, all stems of hips were in clinical neutral alignment and the prostheses survivorship rates for all-causes revisions was $97.6 \%$.

Conclusion: Modular THA is a valuable alternative to render favorable outcomes for treatment of osteoarthritis secondary to severe coxa vara.

Keywords: osteoarthritis, coxa vara, modular, total hip arthroplasty

\section{Introduction}

Coxa vara is a hip disorder characterized by varus deformity of the proximal femur. ${ }^{1-4}$ Congenital coxa vara is rare, with an estimated incidence of 1 in $25,000 .{ }^{1}$ In addition, coxa vara could be a sequela resulting from avascular necrosis after the treatment of developmental hip dysplasia (DDH), Legg-Calve-Perthes (LCP) disease, previous femoral osteotomy, childhood septic arthritis or metabolic bone disease. ${ }^{1-3,5,6}$ Coxa vara is commonly associated with a spectrum of pathologies that involve a short neck, relative overgrowth of the greater trochanter (GT), insufficiency of hip abductors and limb length discrepancy (LLD). ${ }^{1,3,4,7}$ All these deformities in adult patients could lead to osteoarthritis of the hip and degenerations of the spinopelvic complex, which will eventually require total hip arthroplasty (THA). ${ }^{2,6,7}$ On account of the native femoral proximal morphology of these patients, it is technically difficult to restore the normal structure. ${ }^{1,3,6-9}$ A conventional cementless tapered stem tends to be inserted in a varus position, ${ }^{4,10}$ which could lead to a higher distal strain distribution, femoral cortical hypertrophy, thigh pain and negative survivorship in the long term. ${ }^{3,4,6,10}$ To maintain neutral stem alignment, it is recommended in some literatures performing the greater trochanteric osteotomy and trochantoplasty, while both have potential negative 
consequences. $^{1,3,5,6}$ In addition, monoblock stems with a varus neck, hip resurfacing or even customized implants have been given consideration. ${ }^{2,11}$ However, all of the above methods have limited capacity in adjusting femoral offset (FO), anteversion and length of lower limbs. In contrast, the modular THA allows adjustment of the abnormal femoral anteversion and offset of coxa vara and versatility to optimize soft tissue tension to improve biomechanics, appropriately matching the differences of the medullary cavity to generate maximal stability and protecting the abductor tendon insertion. ${ }^{2,12-14}$ To our knowledge, there has been no published study analyzing the results of modular THA in the treatment of deformities of coxa vara. Therefore, the aim of this study was to evaluate early- to mid-term postoperative outcomes of treating coxa vara, stem position, complications and further survivorship by modular THA.

\section{Methods}

\section{Patients}

We retrospectively reviewed 62 patients in our institution who underwent primary THA from May 2008 to December 2019 for the treatment of osteoarthritis secondary to coxa vara after the institutional review board of our hospital approval. The patients were diagnosed by the radiographic criterion of decreased neck-shaft angle (NSA). ${ }^{1,3}$ The exclusion criteria were 1) THA without a modular cementless prosthesis, 2) previous greater trochanteric osteotomy or trochantoplasty, 3) follow-up time of less than one year, or 4) patients with inadequate clinical or radiographic data. Therefore, a total of 33 patients (42 hips) were ultimately included in this study. The demographics and clinical information were based on the historical and clinical findings in medical records (Table 1). The reasons for coxa vara included DDH $(n=20)$, osteonecrosis of the femoral head $(n=6)$, septic arthritis $(n=9)$, Legg-Calve-Perthes disease $(n=3)$ and deformities after trauma $(n=4)$. According to the Bucholz-Ogden (B-O) classification, 6,15 21 hips were classified as B-O Type III, and 21 hips were classified as Type IV by preoperative radiographs.

\section{Surgical Procedure}

All operations were performed by one senior surgeon (YG Zhou) with a posterolateral approach using cementless modular femoral components (S-ROM, DePuy, Warsaw, IN, USA), which consisted of a fluted stem for rotational stability, a distal slot to prevent deformation, and a modular metaphyseal sleeve that adjusted the anteversion. $^{2,13}$ The cementless acetabular component (Pinnacle, DePuy, Warsaw, USA, n=30; Duraloc, DePuy, Warsaw, USA, n=9; Betacup, Link, Hamburg, Germany, $n=3$ ) was utilized. The detailed surgical procedure has been reported in our previous literature. ${ }^{16,17}$ The fascia was divided along the line of the incision and the gluteus maximus was split. The short external rotators were divided close to their femoral attachment. Subsequently, the hip joint was dislocated, and the femoral head was resected. A ceramic-on-ceramic bearing was placed on the cup, which was implanted at the anatomic position. Next following prophylactic cerclage wiring of the femur in osteoporosis cases and reaming, proximal cone reaming of the femur canal was performed until the maximum cortical contact was reached. Then, based on the

Table I Patient Characteristics

\begin{tabular}{|l|c|c|c|c|}
\hline & Total & B-O Type III & B-O Type IV & P-value \\
\hline Number of hips (patients) & $42(33)$ & $21(16)$ & $21(17)$ & $39.4 \pm 10.8$ \\
\hline Age (years)* & $42.3 \pm 13.1$ & $45.2 \pm 14.6$ & $158.2 \pm 9.2$ & 0.253 \\
\hline Height (cm)* & $160.5 \pm 8.9$ & $161.8 \pm 9.1$ & $22.4 \pm 3.3$ & 0.298 \\
\hline BMI (kg/m2)* & $22.26 \pm 3.37$ & $24.5 \pm 5.4$ & $4(24 \%)$ & $13(76 \%)$ \\
\hline Gender & $11(33 \%)$ & $7(44 \%)$ & $76.2 \pm 45.0$ \\
\hline Male & $22(67 \%)$ & $9(56 \%)$ & \\
\hline Female & $69.9 \pm 43.7$ & $63.7 \pm 42.6$ & & 0.360 \\
\hline Follow-up (months)* &
\end{tabular}

Note: *The values are given as the mean and standard deviation. Abbreviation: BMI, body mass index. 
anatomical morphology of the metaphysis, appropriate conical or triangular reaming was performed again to match the sleeve and the metaphysis. With the trial seated in the femur, reduction was performed. A subtrochanteric transverse osteotomy might be performed for femoral shortening if reduction was impossible by measuring the vertical distance between the femoral head and the cup. Eight hips with Crowe IV DDH due to high dislocation underwent subtrochanteric osteotomy after removing the trial. The osteotomy position was planned to approximately $1-2 \mathrm{~cm}$ beneath the lesser trochanter. Prophylactic cerclage wires were placed proximally and distally around the planned fragment. Next a transverse osteotomy was performed and the length of the osteotomy was based on the distance measured before, leaving about $1-1.5 \mathrm{~cm}$ with surgeon's discretion. The wires were tightened and fixed to make the end achieve maximum contact. Then trial reduction was performed again. This step could be repeated until reduction was finished. After completion of trial reduction and the evaluation of stability, equal limb length and soft tissue tension by adjusting the head/neck length, the femoral component was implanted. Finally, femoral anteversion was modified with the advantages of a modular prosthesis to maintain the tension of the gluteus medius. All patients were advised to partial weight-bearing exercise and walk with the aid of two crutches for six weeks on the second day after the operation.

\section{Clinical Evaluation}

Routine follow-ups were scheduled at three, six, and twelve months and every year thereafter. Patients who had not returned for follow-up were contacted by telephone and were asked to return to the clinic. The final follow-up evaluation was performed at an average of 69.9 \pm 43.7 months (range, 15 to 153 months) postoperatively.

Clinical evaluation was performed using the Harris hip scoring (HHS), ${ }^{18-20}$ leg length discrepancy (LLD), and limp preoperatively and at the final follow-up. The measurement of LLD is defined as the difference in vertical distance between the lower margin of the lesser trochanter and the line of connecting intertear drops. ${ }^{8,9,21,22}$ All the patients were adults with no bony growth further and no differences in tibial length of lower limbs, which ensured that LLD just reflected the changes on hips. Any visual manifestation of lateral imbalance of the pelvic movement during gait was identified as the limp. Limp was categorized as none, slight, moderate, severe, or unable to walk. $^{2,3,11}$

\section{Radiographic Assessment}

Anteroposterior (AP) radiographs of the pelvis preoperatively and at the final follow-up were analyzed, all of which were obtained in the supine position using a standardized instruments and procedures to achieve reproducible projection.

The following parameters were measured in AP radiographs (Figure 1): Greater trochanteric height (GTH) $(\mathrm{mm}):^{1-4,7}$ the perpendicular distance between the center of rotation(COR) of hip and the tip of the trochanter; femoral offset $(\mathrm{FO})(\mathrm{mm}):^{2,8,21}$ the perpendicular distance from the COR to the femoral medullary central axis; abductor lever arm (ALA) (mm): $:^{2,3,21}$ the perpendicular distance between the COR and the abductor force vector; body weight lever arm (BWLA) (mm): ${ }^{2,3,21}$ the perpendicular distance between the COR and the body weight vector. Femoral stem alignment was also evaluated, which was determined by measuring the angle between the longitudinal axis of the stem and the longitudinal axis of the femur. The stem alignment was classified as neutral, valgus $\left(>3^{\circ}\right.$ of lateral deviation), or varus $\left(>3^{\circ}\right.$ of medial deviation). ${ }^{4,10,22}$

Implant fixation and osteolysis around the acetabular component (according to Latimer and Lachiewicz ${ }^{23}$ ) and the femoral component (according to Engh et $\mathrm{al}^{24}$ ) were evaluated by comparing AP radiographs at the final follow-up to those at post-operation. The lesions were located according to the 3 zones on the acetabular side, which are described by DeLee and Charnley. ${ }^{25}$ Heterotopic ossification was evaluated according to Brooker et al. ${ }^{26}$ Complications, reoperation and implant survival rates were also evaluated.

All radiographic measurements and classification were made by two independent investigators using the digital technique of Digimizer v5.4 (MedCalcSoftware, Ostend, Belgium) to eliminate magnification error. All of the parameters were measured again four weeks later without knowledge of the previous results to assess the intraobserver reliability. These intraclass correlation coefficients (ICCs) for interobserver (0.82 to 0.91$)$ and intraobserver (0.81 to 0.88 ) reliability indicated high reproducibility.

\section{Statistical Analysis}

The mean and standard deviation were calculated for continuous variables in each group. The normal distribution of all data was checked by the Shapiro-Wilk and Kolmogorov-Smirnov test. Student's $t$-test was used to 

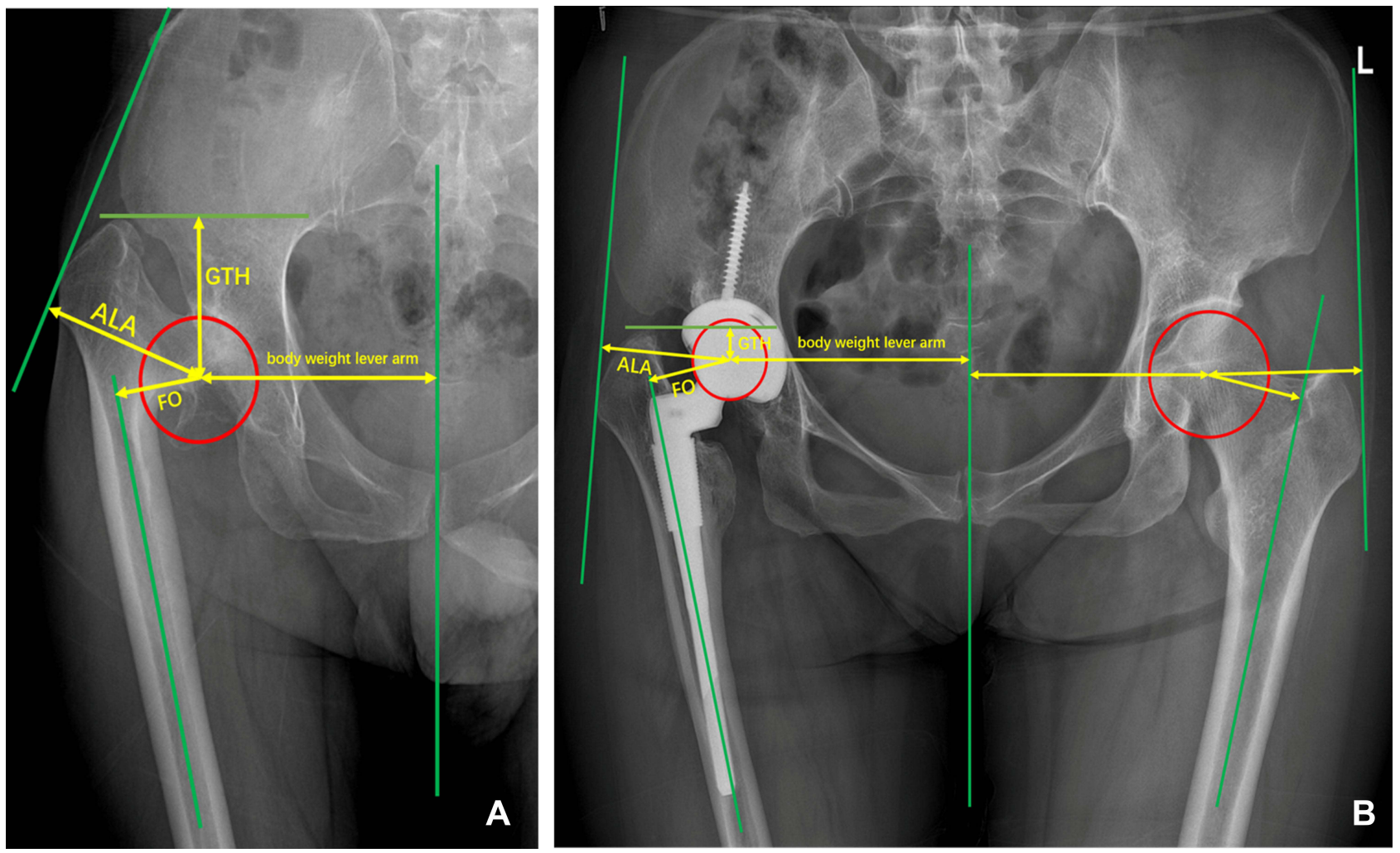

Figure I Diagrams for radiographic measurement of coxa vara (A) pre-operation; (B) the final follow-up.

assess the significance on mean parameter values. KaplanMeier analysis was performed to determine the probability of survivorship. A P-value of $<0.05$ was considered significant. All analyses were performed using SPSS version 26.0 (IBM, Armonk, NY, USA).

\section{Results}

The mean operation time was $3.47 \pm 1.51$ hours (range, 1.58 to 7.67), and the mean amount of estimated blood loss was $542.42 \pm 467.89 \mathrm{~mL}$ (range, 100 to 2600 ) for all patients.

\section{Clinical Evaluation}

Clinically, HHS in pre-operation and final follow-up improved significantly $(\mathrm{p}<0.001)$ on average from 42.90 \pm 14.44 points to $89.54 \pm 4.75$ points. Patients demonstrated diminished or no limping in $88 \%$ (29/33) of hips. In all patients, seven (21\%) had slight limps, and six (18\%) had moderate limps compared with all had moderate to severe limp preoperatively. Most of the limbs in the operation were prolonged, and the mean LLD decreased from 33.3 $\pm 19.4 \mathrm{~mm}$ to $5.0 \pm 5.8 \mathrm{~mm}(\mathrm{p}<0.001)$. Twenty-seven patients $(82 \%)$ thought that total equality of the lower limbs was obtained compared to the preoperative state in their own perceptions. Six of the LLDs resulted from unrecovered deformity of the spinopelvic oblique, and two explained that the LLDs resulted from insufficient exercise postoperatively, causing poor strength of muscle (Table 2).

\section{Radiographic Assessment}

For all radiographic parameters, the GTH and BWLA were significantly decreased compared to those preoperatively, and the FO and ALA were significantly increased (Table 3 and Figure 2). All stem of hips measured at the final follow-up were in clinical neutral alignment (varus 0.87 $\pm 1.12^{\circ}$ on average) between the longitudinal axis of the stem and the longitudinal axis of the femur.

Radiographic analysis at the final follow-up demonstrated that all prostheses were ingrown and were in stable positions. Two hips had a slight osteolysis in zone 1 and three hips had a slight osteolysis in zone 2 of the

Table 2 Long-Term Clinical Evaluation

\begin{tabular}{|l|c|c|c|}
\hline & Pre-Operation & Final Follow-Up & P-value \\
\hline HHS (points) & $42.9 \pm 14.4$ & $89.5 \pm 4.8$ & $<0.001$ \\
\hline LLD (mm) & $33.3 \pm 19.4$ & $5.0 \pm 5.8$ & $<0.001$ \\
\hline
\end{tabular}

Note: The values are given as the mean and standard deviation.

Abbreviations: HHS, Harris hip score; LLD, limb length discrepancy. 
Table 3 Radiographic Assessment

\begin{tabular}{|l|c|c|c|}
\hline & $\begin{array}{c}\text { Pre- } \\
\text { Operation }\end{array}$ & $\begin{array}{c}\text { Final Follow- } \\
\text { Up }\end{array}$ & P-value \\
\hline GTH $(\mathrm{mm})$ & $37.2 \pm 12.0$ & $3.4 \pm 8.3$ & $<0.001$ \\
\hline FO $(\mathrm{mm})$ & $23.1 \pm 7.8$ & $31.5 \pm 5.9$ & $<0.001$ \\
\hline Body weight lever arm $(\mathrm{mm})$ & $102.9 \pm 10.6$ & $84.8 \pm 9.0$ & $<0.001$ \\
\hline ALA $(\mathrm{mm})$ & $48.8 \pm 9.7$ & $53.1 \pm 9.8$ & 0.014 \\
\hline
\end{tabular}

Note: The values are given as the mean and standard deviation.

Abbreviations: GTH, great trochanteric height; FO, femoral offset; ALA, abductor lever arm.

acetabulum. No loosening or ossification was observed (Figure 3). Bony union was achieved for all patients undergoing subtrochanteric osteotomy in 6 months postoperatively.

\section{Complications}

Periprosthetic fracture of the distal femur occurred in one hip eight days after the operation owing to severe osteoporosis and excessive exercise, which was treated by open reduction with cable cerclage wiring immediately and healed in six months. One hip underwent revision because of loosening of the femoral component 5.2 years after the operation, which demonstrated a satisfactory outcome at the final follow-up. There were no other complications, such as nerve injury, dislocation or infection. With revision for any reason as the end point, the implant survival of $97.6 \%$ at the final follow-up was observed by KaplanMeier survival analysis (Figure 4).

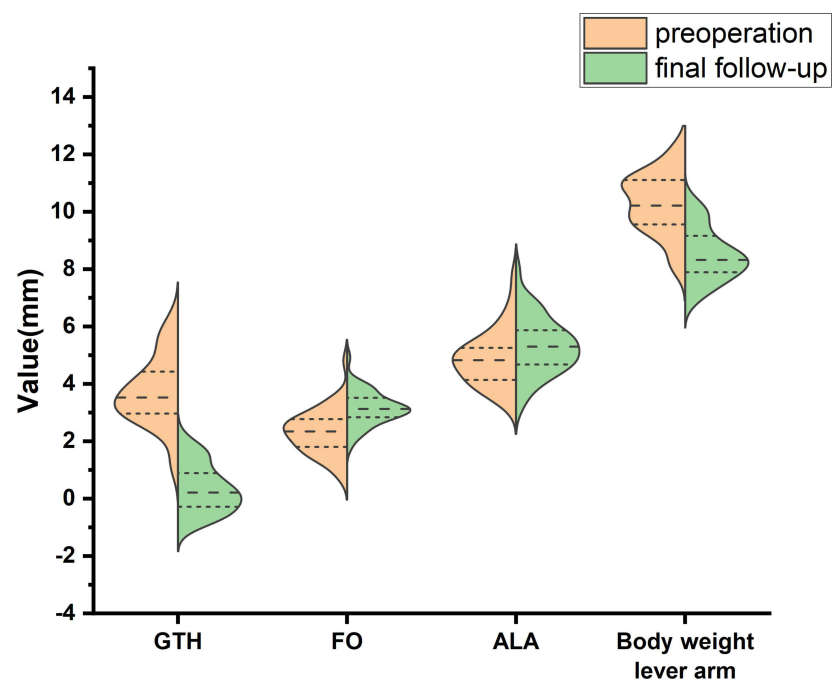

Figure 2 Scattergram showed there were significant differences in radiographic parameters (GTH, FO, ALA and Body weight lever arm) between the pre-operation and the final follow-up.

\section{Discussion}

The treatment of osteoarthritis secondary to coxa vara, which is associated with low NSA, overgrowth of the GT and limp, causing pelvic obliquity and degeneration of the lumbar spine, is challenging for surgeons. ${ }^{3,8,11}$ Many methods have been proposed for the restoration of normal anatomic structure and improvement of biomechanics, ${ }^{2,3,6,10,27}$ while little attention has been given to modular THA. We demonstrated that the outcomes of modular THA in patients with coxa vara who were followed up for more than 5 years were inspiring and positive, which achieved the expected satisfaction for all patients.

Customed cementless tapered stems have been utilized for years, but they may tend to varus on account of the native proximal morphology of the femur, for dictating the best fit of the prosthesis. For the Corail stem, mild varus alignment does not seem to have a negative effect, as Vidalain and Boldt reported. ${ }^{4,10,28}$ However, techniques to maintain a neutral alignment, such as lowering the neck cut and lateralizing the entry point to the edge of GT, still risked damaging the abductor, altered the required offset or best fit of the prosthesis, and affected length of lower limbs and stability, meaning that varus alignments were not the original goal but were the result of compromise, let alone the disadvantages mentioned above. ${ }^{4,28,29}$ A requirement for a more tailored prosthesis with either increased modularity or a custom-made prosthesis is feasible, while the latter is costly and involves a timeconsuming process. ${ }^{11}$

Some scholars have suggested that greater trochanteric osteotomy or trochantoplasty is necessary for neutral alignment and avoiding obstruction of the GT, as Bartoníbek et $\mathrm{al}^{6}$ and Yoo et $\mathrm{al}^{3}$ reported. However, these techniques add to the complexity of the operation and the opportunity for infection owing to the increased blood loss, the higher frequency of taking intraoperative radiographs and the delay of rehabilitation, associated with femoral fracture and osteotomy nonunion. ${ }^{2,3,6,11}$ Bartoníbek et al reported the result of only valgus intertrochanteric osteotomy in 15 patients with coxa vara, and $20 \%$ of patients had already undergone conversion to THA at the final follow-up because of the poor surgical technique. Refixation of osteotomies is also highly technically demanding. Although the procedure has been improved, ${ }^{5}$ certain complications, high rates of dissatisfaction may be unavoidable. ${ }^{1,5,6}$ Roberts et $\mathrm{al}^{1}$ reported patients with congenital coxa vara by femoral valgus derotational osteotomy suggested $22 \%$ recurrence rate, resulting in residual deformity and progression to 

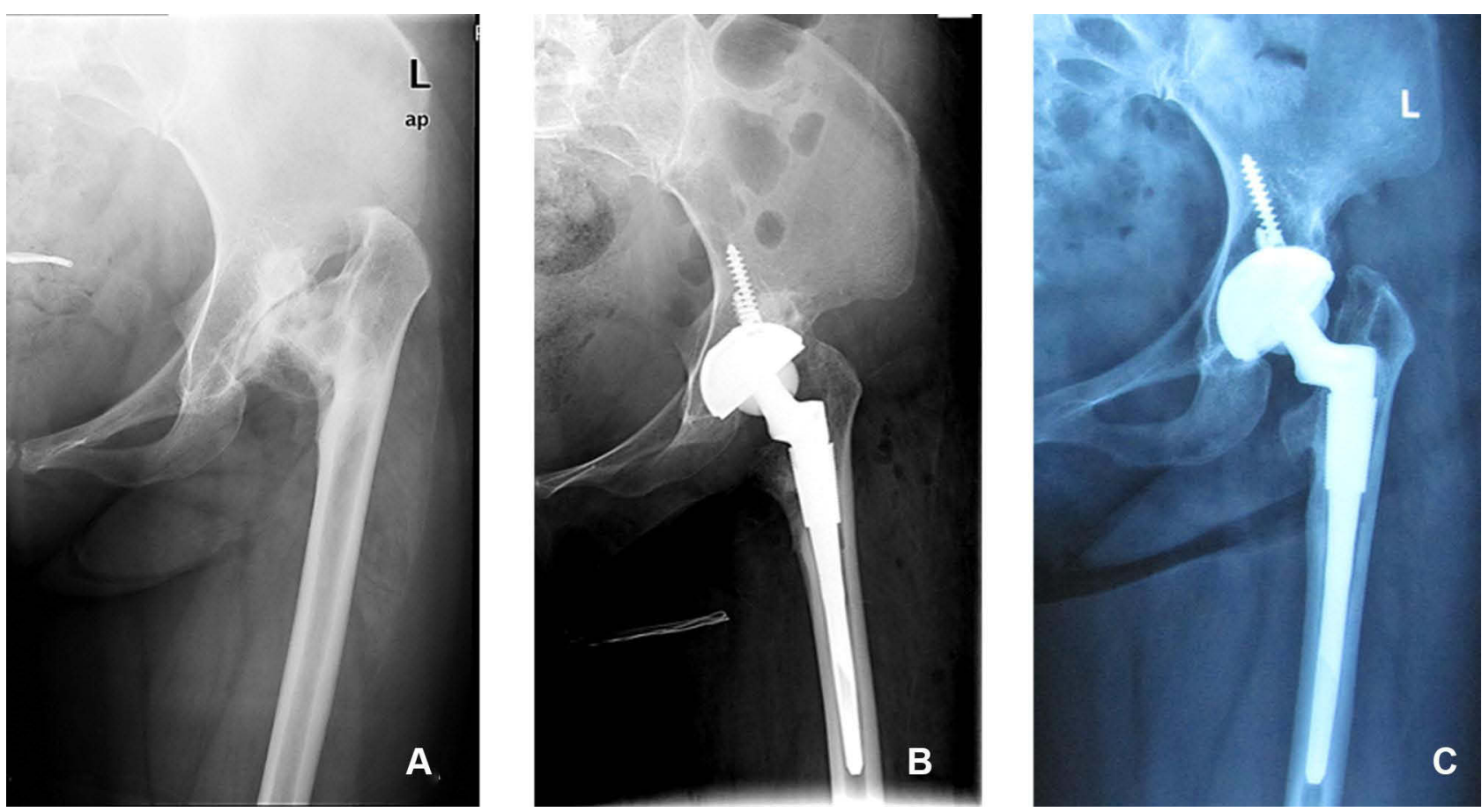

Figure 3 A 39-year-old woman with severe coxa vara. Preoperative (A) anteroposterior X-ray showed the severe deformity of the hip. Postoperative (B) anteroposterior X-ray showed normal anatomical structure and biomechanics were restored and the modular prosthesis achieved the initial stability. ${ }^{37}$ (C) After II.2 years it showed the optimal positions of the acetabular cup and femoral stem and the patient had no related symptoms.

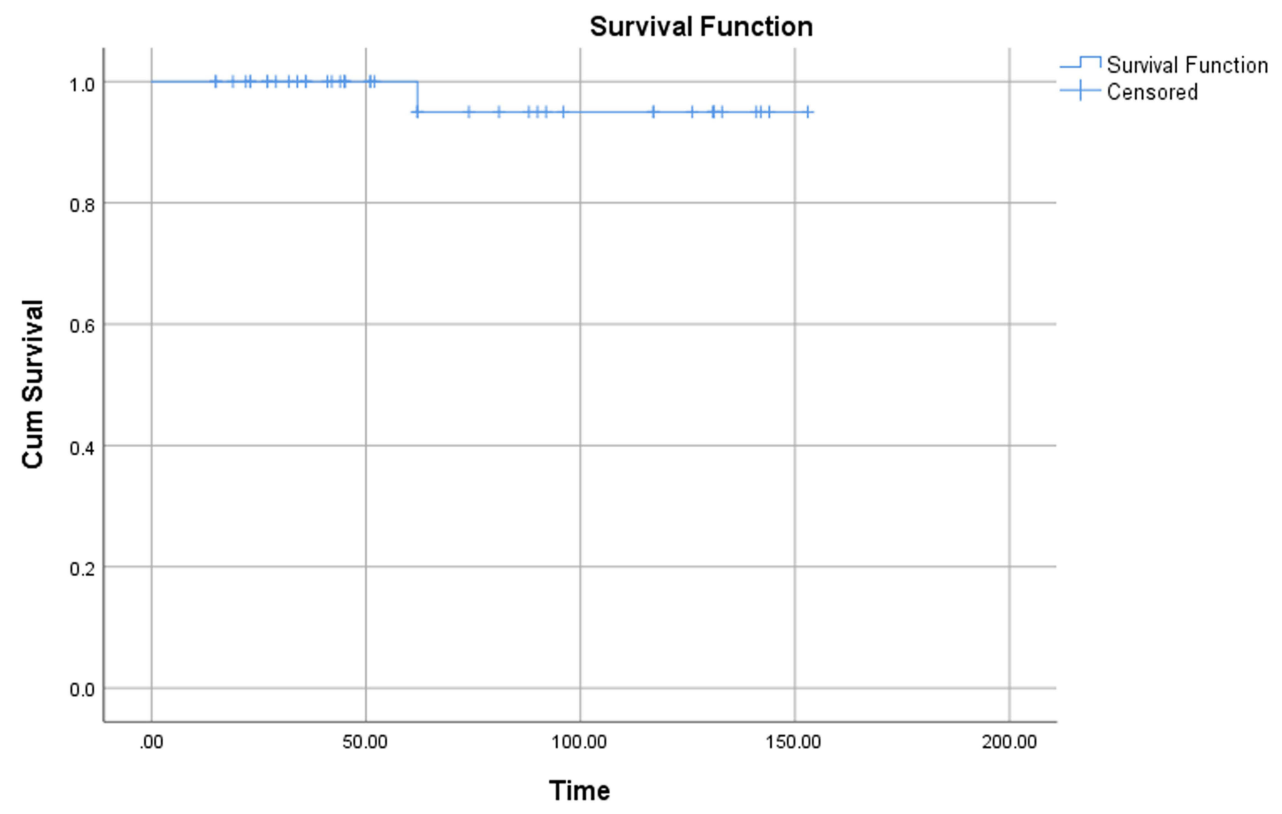

Figure 4 The Kaplan-Meier survival curve with revision for any reason as the end point was shown.

osteoarthritis over time, which indirectly reflected that severe osteoarthritis could be the final result regardless of whether the osteotomy was performed. In addition, THA performed after failed trochanteric osteotomies, including trochantoplasty, is also known to be of increased operating time and blood loss, more technical difficulties and higher complication rates $(6 \%-31 \%){ }^{30-33}$ Osawa et $\mathrm{al}^{32}$ reported that the HHS after THA at the final follow-up was significantly poorer in the osteotomy group than in the primary group due to poor physical function caused by osteotomy, 
and Carlile et $\mathrm{al}^{27}$ suggested there were higher restrictions on patient selection and the surgical techniques for resurfacing arthroplasty in coxa vara. More complicated arthritic hips, such as hips with large cysts, osteopenia, LLD $>1 \mathrm{~cm}$ or a diminished FO, that usually occurs in coxa vara, may be better served by a modular THA. ${ }^{27,34,35}$

Compared with the methods discussed above, the distinct advantages of modular THA depend on individualization for coxa vara. First, the modularity of the SROM allowed relatively free adjustment of the abnormal femoral anteversion by rotation between the stem and sleeve to avoid the obstruction of the overhanging greater trochanter, protecting the abductor tendon insertion and decreasing instability. ${ }^{2,12,14}$ Second, different sizes of sleeves and stems could appropriately match the different morphologies of the medullary cavity to generate maximal contact, which decreased the occurrence of proximal bone loss due to stress shielding, ${ }^{13,14}$ and alter the FO and length to achieve strong initial fixation. As a decrease in FO is common due to a stem NSA became greater than the native value after THA, ${ }^{8,21}$ an increased offset is necessary during surgery to reduce the load of the abductor, which has been showed in our radiographic results, and it decreased the joint reaction force, improving limp and enhancing the survival of the artificial join. The significant decrease in the mean LLD represented not only that LLD was addressed but also that the effective ALA was increased by the lengthening of the affected limb with modular THA, which is beneficial to biomechanics. ${ }^{2,21,36}$ In addition, the decrease in GTH manifested the COR was restored in normal morphology. All stems were in varus at an average of $0.87 \pm 1.12^{\circ}$ but in clinical neutral alignment, meaning that a slight varus position $\left(<3^{\circ}\right)$ was acceptable.

Another advantage not to be ignored is the use of conical sleeves to obtain stability due to the narrow and straight shape of the medullary cavity in coxa vara. ${ }^{1,4,8}$ It is difficult for tapered stems to sink and stabilize deeply, which not only risks fracture of the metaphysis but also leads to the dilemma of reduction. The stem with conical sleeve could sink deeper and varus alignment is avoided. It has been reported that subtrochanteric osteotomy is usually necessary for some high dislocation hips such as Crowe type IV DDH. ${ }^{13,16,37}$ However, in our study, some of these varus hips might avoid osteotomy by matching the metaphyseal with a conical sleeve, benefiting from the deeper implantation of stems, which is worthy of further study (Figure 5).

Kang et al ${ }^{14}$ demonstrated similar good mid-term results in secondary coxarthrosis, including dysplastic hip or deformed femur, by the S-ROM prosthesis, which supported our results, while radiological parameters in this study were more specific and comprehensive. Seufert et $\mathrm{al}^{2}$ also reported outcomes for the arthritis with LCP disease with modular THA. However, the author did not demonstrate signs of varus in their cases, and the sample size was small. Abouelela et $\mathrm{al}^{38}$ and Talmo et $\mathrm{al}^{12}$ studied modular THA after failed internal fixation of trochanteric fracture, $5 \%$ to $11 \%$ malunion of which caused varus deformity. Abouelela et al did not emphasize whether there was varus in their cases, and the study by Talmo et al is just case reports.
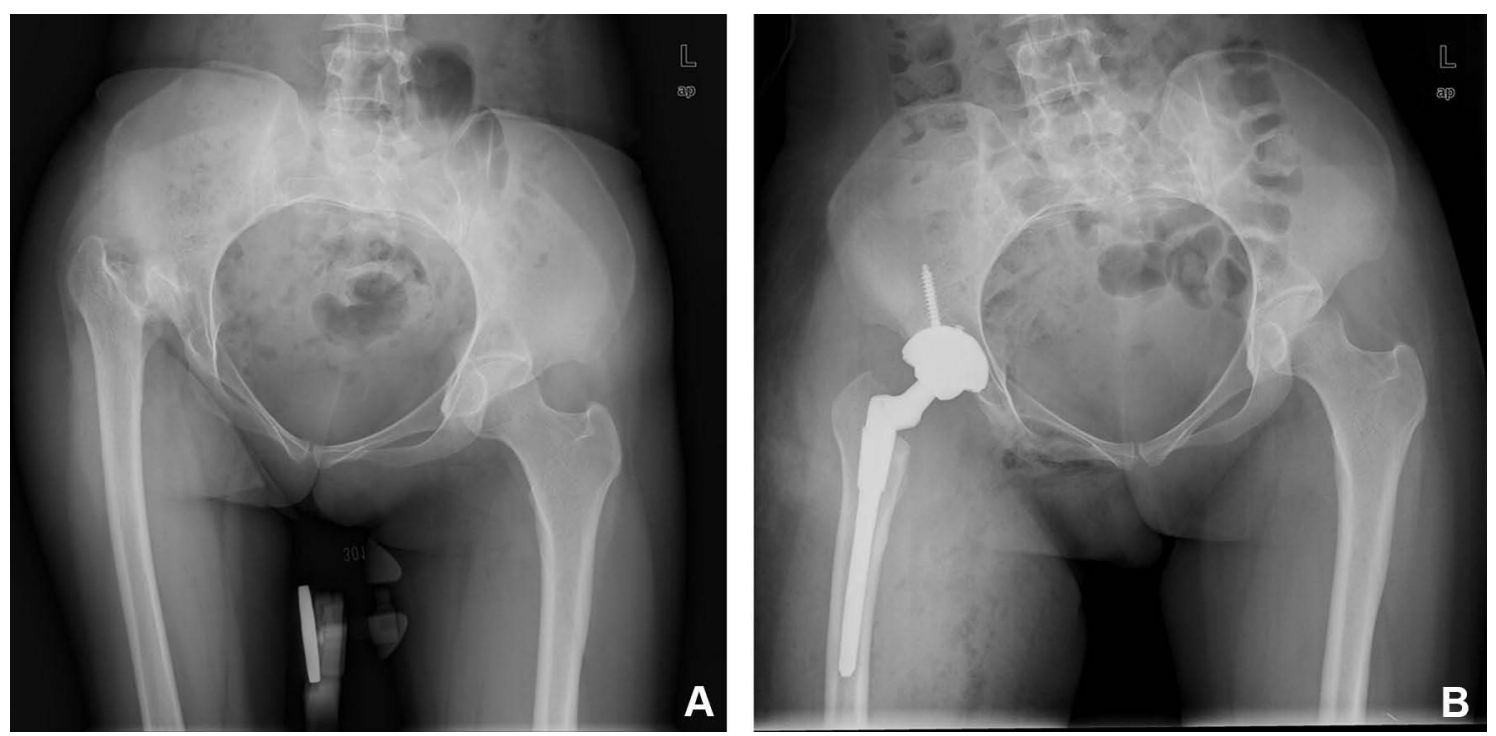

Figure 5 Preoperative $(\mathbf{A})$ the deformity of coxa vara is associated with a high dislocation and a narrow and straight medullary cavity, which might be undergone the subtrochanteric osteotomy. Postoperative (B) the use of conical sleeve in SROM prosthesis made the stem sink deeper to avoid the osteotomy probably. 
This study has several limitations. First, it is a retrospective, single-center review based on a relatively small sample size. However, coxa vara is a rare condition, and a large-cohort prospective study is hard to perform. Our patients were identified from a consecutive series, reducing the possibility of selection bias. Second, patients with bilateral coxa vara were not excluded and this could influence the results slightly. Moreover, we included patients affected by coxa vara due to different reasons, while all of hips had similar morphological features and solutions. Third, our study was performed in East Asia. Our results might not be generalizable to patients with a higher body mass index in western countries. Fourth, the measures of some parameters in this study were indirect, while we believe good medium-term outcomes lend credibility to modular THA and may serve as a basis for further research on more modular prostheses in longerterm follow-ups.

\section{Conclusion}

In conclusion, we believe that modular THA is effective and promising for osteoarthritis secondary to severe coxa vara caused by multiple primary diseases, which simplifies the operation, improves the deformity of hips, avoids unnecessary complications and achieves positive and satisfactory outcomes. However, larger comparative studies with more types of prostheses and longer follow-ups are required to validate this technique.

\section{Ethical Approval}

This retrospective review study involving human participants was in accordance with the ethical standards of the institutional and national research committee and with the 1964 Helsinki Declaration and its later amendments or comparable ethical standards. This study was approved by the medical ethics committee of Chinese PLA General Hospital.

\section{Informed Consent}

Prior to the study, the written informed consent was obtained from all patients for publication of this study and any accompanying images.

\section{Acknowledgments}

The authors thank all staff from the participating departments and clinics.

\section{Funding}

This research did not receive any specific grant from funding agencies in the public, commercial, or not-forprofit sectors.

\section{Disclosure}

The authors report no conflicts of interest in this work. Bohan Zhang and Jingyang Sun contributed equally to this work and are considered co-first authors for this study.

\section{References}

1. Roberts DW, Saglam Y, De La Rocha A, Frasquillo BN, TulchinFrancis K, Kim HKW. Long-term outcomes of operative and nonoperative treatment of congenital coxa vara. $J$ Pediatr Orthop. 2018;38(4):193-201. doi:10.1097/BPO.0000000000000782

2. Seufert CR, McGrory BJ. Treatment of arthritis associated with legg-calve-perthes disease with modular total hip arthroplasty. J Arthroplasty. 2015;30(10):1743-1746. doi:10.1016/j.arth.2015.04.025

3. Yoo JI, Parvizi J, Song JU, Ha YC, Lee YK, Koo KH. Trochantoplasty for total hip arthroplasty in patients with coxa vara deformity. J Arthroplasty. 2017;32(7):2199-2203. doi:10.1016/j. arth.2017.02.010

4. Murphy CG, Bonnin MP, Desbiolles AH, Carrillon Y, Aït Si Selmi T. Varus will have varus; a radiological study to assess and predict varus stem placement in uncemented femoral stems. Hip Int. 2016;26 (6):554-560. doi:10.5301/hipint.5000412

5. Hefti F, Donnan L, Krieg AH. Treatment of shepherd's crook deformity in patients with polyostotic fibrous dysplasia using a new type of custom made retrograde intramedullary nail: a technical note. J Child Orthop. 2017;11(1):64-70. doi:10.1302/ 1863-2548.11.170002

6. Bartonicek J, Vavra J. Valgus intertrochanteric osteotomy for coxa vara of Bucholz-Ogden Types II and III in patients older than 30 years. Arch Orthop Trauma Surg. 2011;131(9):1211-1217. doi:10.1007/s00402-011-1278-5

7. Haversath M, Busch A, Jager M, Tassemeier T, Brandenburger D, Serong S. The 'critical trochanter angle': a predictor for stem alignment in total hip arthroplasty. J Orthop Surg Res. 2019;14(1):165. doi:10.1186/s13018-019-1206-x

8. Boisgard S, Descamps S, Bouillet B. Complex primary total hip arthroplasty. Orthop Traumatol Surg Res. 2013;99(1 Suppl):S34-42. doi:10.1016/j.otsr.2012.11.008

9. Dastane M, Dorr LD, Tarwala R, Wan Z. Hip offset in total hip arthroplasty: quantitative measurement with navigation. Clin Orthop Relat Res. 2011;469(2):429-436. doi:10.1007/s11999-010-1554-7

10. Khalily C, Lester DK. Results of a tapered cementless femoral stem implanted in varus. J Arthroplasty. 2002;17(4):463-466. doi:10.1054/ arth.2002.32171

11. Kim JT, Kim HS, Lee YK, Ha YC, Koo KH. Total hip arthroplasty with trochanteric ostectomy for patients with angular deformity of the proximal femur. J Arthroplasty. 2020;35(10):2911-2918. doi:10.1016/j.arth.2020.04.099

12. Talmo CT, Sambaziotis C, Bono JV. Conversion hemiarthroplasty and valgus osteotomy after failed ORIF of hip intertrochanteric fractures. Orthopedics. 2013;36(9):693-696. doi:10.3928/0147744720130821-04

13. Ma H-Y, Lu Q, Sun J-Y, et al. One-Stage total hip arthroplasty with modular S-ROM stem for patients with bilateral Crowe Type IV developmental dysplasia. Orthop Surg. 2020;12(6):1913-1922. doi:10.1111/os.12843 
14. Kang JS, Moon KH, Kim RS, Park SR, Lee JS, Shin SH. Total hip arthroplasty using S-ROM prosthesis for dysplastic hip. Yonsei Med J. 2011;52(4):655-660. doi:10.3349/ymj.2011.52.4.655

15. Roposch A, Wedge JH, Riedl G. Reliability of Bucholz and Ogden classification for osteonecrosis secondary to developmental dysplasia of the hip. Clin Orthop Relat Res. 2012;470(12):3499-3505. doi:10.1007/s11999-012-2534-x

16. Sun J, Zhang G, Shen J, et al. Dislocation height performs well in predicting the use of subtrochanteric osteotomy in Crowe Type IV hips. Ther Clin Risk Manag. 2020;16:989-997. doi:10.2147/TCRM. S272771

17. Zhou Y, Sun C, Wang Y. New method addressing the problem of using ceramic-on-ceramic bearing in too small acetabulum of high-riding DDH patients with THA. Semin Arthroplasty. 2012;23 (4):226-231. doi:10.1053/j.sart.2012.12.006

18. Shen J, Sun J, Ma H, Du Y, Li T, Zhou Y. High Hip center technique in total hip arthroplasty for Crowe Type II-III developmental dysplasia: results of midterm follow-up. Orthop Surg. 2020;12 (4):1245-1252. doi:10.1111/os.12756

19. Erivan R, Villatte G, Ollivier M, Paprosky WG. Painful hip arthroplasty: what should we find? Diagnostic approach and results. $J$ Arthroplasty 2019;34(8):1802-1807. doi:10.1016/j.arth.2019.04.014

20. Harris WH. Traumatic arthritis of the hip after dislocation and acetabular fractures: treatment by mold arthroplasty. An end-result study using a new method of result evaluation. J Bone Joint Surg Am. 1969;51(4):737-755. doi:10.2106/00004623-196951040-00012

21. Flecher X, Ollivier M, Argenson JN. Lower limb length and offset in total hip arthroplasty. Orthop Traumatol Surg Res. 2016;102(1 Suppl):S9-20. doi:10.1016/j.otsr.2015.11.001

22. Lee YK, Kim JT, Alkitaini AA, Kim KC, Ha YC, Koo KH. Conversion hip arthroplasty in failed fixation of intertrochanteric fracture: a propensity score matching study. J Arthroplasty. 2017;32 (5):1593-1598. doi:10.1016/j.arth.2016.12.018

23. Latimer HA, Lachiewicz PF. Porous-coated acetabular components with screw fixation. Five to ten-year results. J Bone Joint Surg Am. 1996;78(7):975-981. doi:10.2106/00004623-199607000-00001

24. Engh CA, Glassman AH, Suthers KE. The case for porous-coated hip implants. The femoral side. Clin Orthop Relat Res. 1990;261:63-81.

25. DeLee JG, Charnley J. Radiological demarcation of cemented sockets in total hip replacement. Clin Orthop Relat Res. 1976;(121):20-32.

26. Brooker AF, Bowerman JW, Robinson RA, Riley LH. Ectopic ossification following total hip replacement. Incidence and a method of classification. J Bone Joint Surg Am. 1973;55(8):1629-1632. doi:10.2106/00004623-197355080-00006
27. Carlile GS, Wakeling CP, Fuller N, Fern D, Norton MR. Hip resurfacing arthroplasty in patients with varus deformity of the femoral neck-shaft angle. Hip Int. 2011;21(2):225-230. doi:10.5301/ hip. 2011.6498

28. Vidalain JP. Twenty-year results of the cementless Corail stem. Int Orthop. 2011;35(2):189-194. doi:10.1007/s00264-010-1117-2

29. Vresilovic EJ, Hozack WJ, Rothman RH. Radiographic assessment of cementless femoral components. Correlation with intraoperative mechanical stability. $J$ Arthroplasty. 1994;9(2):137-141. doi:10.1016/0883-5403(94)90062-0

30. Kawasaki M, Hasegawa Y, Sakano S, Masui T, Ishiguro N. Total hip arthroplasty after failed transtrochanteric rotational osteotomy for avascular necrosis of the femoral head. J Arthroplasty. 2005;20 (5):574-579. doi:10.1016/j.arth.2005.01.018

31. Shigemura T, Yamamoto Y, Murata Y, et al. Total hip arthroplasty after failed transtrochanteric rotational osteotomy for osteonecrosis of the femoral head: a systematic review and meta-analysis. Orthop Traumatol Surg Res. 2018;104(8):1163-1170. doi:10.1016/j. otsr.2018.06.019

32. Osawa Y, Seki T, Morita D, Takegami Y, Okura T, Ishiguro N. Total hip arthroplasty after transtrochanteric rotational osteotomy for osteonecrosis of the femoral head: a mean 10-year follow-up. J Arthroplasty. 2017;32 (10):3088-3092. doi:10.1016/j.arth.2017.05.020

33. Gerundini M, Avai A, Taglioretti J. Total hip replacement after intertrochanteric osteotomy. Int Orthop. 1995;19(2):84-85. doi:10.1007/BF00179965

34. Silva M, Lee KH, Heisel C, Dela Rosa MA, Schmalzried TP. The biomechanical results of total hip resurfacing arthroplasty. $J$ Bone Joint Surg Am. 2004;86(1):40-46. doi:10.2106/00004623-200401000-00007

35. Costa CR, Johnson AJ, Naziri Q, Mont MA. Review of total hip resurfacing and total hip arthroplasty in young patients who had Legg-Calve-Perthes disease. Orthop Clin North Am. 2011;42 (3):419-422,viii. doi:10.1016/j.ocl.2011.04.002

36. Houcke JV, Khanduja V, Pattyn C, Audenaert E. The history of biomechanics in total hip arthroplasty. Indian J Orthop. 2017;51 (4):359-367. doi:10.4103/ortho.IJOrtho_280_17

37. Wang S, Zhou Y, Ma H, Du Y, Piao S, Wu W. Mid-term results of total hip replacement with subtrochanteric osteotomy, modular stem, and ceramic surface in Crowe IV hip dysplasia. Arthroplast Today. 2018;4(3):363-369. doi:10.1016/j.artd.2017.07.003

38. Abouelela AA. Salvage of failed trochanteric fracture fixation using the Revitan curved cementless modular hip arthroplasty. J Arthroplasty. 2012;27(7):1382-1388. doi:10.1016/j.arth.2011.10.021
Therapeutics and Clinical Risk Management

\section{Publish your work in this journal}

Therapeutics and Clinical Risk Management is an international, peerreviewed journal of clinical therapeutics and risk management, focusing on concise rapid reporting of clinical studies in all therapeutic areas, outcomes, safety, and programs for the effective, safe, and sustained use of medicines. This journal is indexed on PubMed Central, CAS,
EMBase, Scopus and the Elsevier Bibliographic databases. The manuscript management system is completely online and includes a very quick and fair peer-review system, which is all easy to use. Visit http://www.dovepress.com/testimonials.php to read real quotes from published authors. 